\title{
Droplet under confinement: Competition and coexistence with a soliton bound state
}

\author{
Xiaoling Cui ${ }^{1,2, *}$ and Yinfeng Ma $\odot^{1,3}$ \\ ${ }^{1}$ Beijing National Laboratory for Condensed Matter Physics, Institute of Physics, Chinese Academy of Sciences, Beijing 100190, China \\ ${ }^{2}$ Songshan Lake Materials Laboratory, Dongguan, Guangdong 523808, China \\ ${ }^{3}$ School of Physical Sciences, University of Chinese Academy of Sciences, Beijing 100049, China
}

(Received 30 October 2020; accepted 1 March 2021; published 19 March 2021)

\begin{abstract}
We study the stability of a quantum droplet and its associated phase transitions in ultracold Bose-Bose mixtures uniformly confined in quasi-two-dimensions with a periodic boundary condition. We show that the confinement-induced boundary effect can be significant when increasing the atom number or reducing the confinement length, which destabilizes the quantum droplet towards the formation of a soliton bound state that has no density modulation along the confined direction. In particular, as increasing the atom number we find the soliton reentrance, while the droplet is stabilized only within a finite number window that sensitively depends on the confinement length. Near the droplet-soliton transitions, they can coexist with each other as two local minima in the energy landscape. Finally, we map out the phase diagram for the droplet-soliton transition and coexistence in the parameter plane of the atom number and confinement length for ${ }^{39} \mathrm{~K}$ boson mixtures.
\end{abstract}

DOI: 10.1103/PhysRevResearch.3.L012027

Introduction. A quantum droplet describes a self-bound many-body state that is stabilized by a quantum effect. It has aroused great attention recently in the field of ultracold atoms, given its successful observation in dipolar gases [1-7] and alkali Bose-Bose mixtures [8-11]. These dilute droplets, as pointed out in a pioneering work by Petrov [12], are stabilized by a subtle balance between the mean-field attraction and the Lee-Huang-Yang (LHY) repulsion from quantum fluctuations. A similar stabilization mechanism has been extended to other droplet systems including Bose-Fermi mixtures [13-18] and dipolar mixtures [19,20].

The stability of a quantum droplet depends crucially on the geometry. In three dimensions (3D), quantum pressure can dissociate the droplet at a small atom number and lead to a liquid-gas transition as observed in experiments [1-11]. In 2D and 1D, the quantum droplet can be supported in quite different interaction regimes as compared to $3 \mathrm{D}$, due to distinct LHY corrections [21]. In this context, it is conceptually important and also practically meaningful to investigate the confinement effect to droplet stability, which can bridge different droplet physics between different geometries. Previously, a few theoretical studies have revealed the significant change in the LHY correction in quasilow dimensions [22-25]. In particular, it was shown that the LHY energy of alkali bosons can gradually change sign to negative as strengthening the confinement [24,25], while the resulting

\footnotetext{
*xlcui@iphy.ac.cn

Published by the American Physical Society under the terms of the Creative Commons Attribution 4.0 International license. Further distribution of this work must maintain attribution to the author(s) and the published article's title, journal citation, and DOI.
}

instability of the droplet and its associated transitions during the dimensional reduction have not been discussed therein.

Apart from the significant change in the LHY correction, we note that the confinement can affect the droplet stability in two other nontrivial ways:

First, it introduces the boundary effect. As illustrated in Fig. 1, for a droplet cloud confined uniformly with welldefined boundaries (central plot), the boundary effect can become significant when the droplet size $\sigma$ is comparable to the trap length $L$, either by increasing the atom number $N$ or by reducing $L$. In either case, the droplet will adjust itself to be compatible with the boundary, which naturally causes instability. Second, the confinement can introduce another channel of a bound state to compete with the droplet. A wellknown example is the bright soliton in quasi-1D (Q1D) that is stabilized by quantum pressure and mean-field attraction [26-28]. In a recent experiment, the droplet-soliton transition was explored in harmonically trapped quasi-1D Bose-Bose mixtures [9], while the confinement effect to qualitatively change the LHY correction was not considered therein.

In this Letter, by fully taking into account the confinement effect, we study the stability of a quantum droplet and its associated transitions in Bose-Bose mixtures confined in Q2D. To clearly see the boundary effect, we consider the uniform trap as depicted in Fig. 1 with a periodic boundary condition. We find that when the boundary effect becomes significant, the droplet becomes unstable and gives way to a soliton bound state that displays no density modulation along the confined direction. As increasing $N$, a soliton to droplet transition occurs at relatively small $N$, while the boundary effect leads to the soliton reentrance at a larger $N$ and thus the droplet can only be stabilized within a finite number window that sensitively depends on the trap length. Near the droplet-soliton transitions, they can coexist with each other as two local minima in the energy landscape. Taking the ${ }^{39} \mathrm{~K}$ 


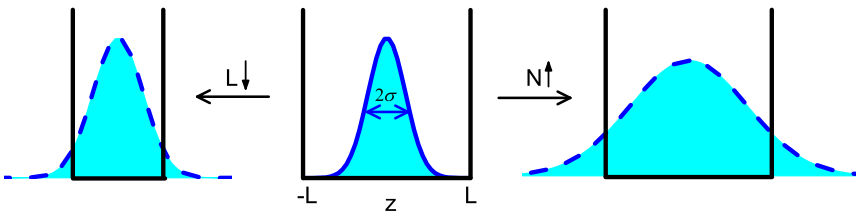

FIG. 1. Boundary effect to a quantum droplet. Starting from a 3D droplet in a uniform trap with a length much larger than the droplet size $L \gg \sigma$ (central plot), the boundary effect can become significant either by increasing the atom number (right) or by reducing the trap length (left). In both cases, we have $L \lesssim \sigma$ and the droplet will encounter instability. Here, the droplet wave function is plotted only along the trap direction.

boson mixture as an example, we have analyzed in detail the droplet-soliton competition and finally mapped out the phase diagram for their transition and coexistence.

Model. The Hamiltonian we consider for the Bose-Bose mixture is $H=\int d \mathbf{r} H(\mathbf{r})$, where $(\hbar=1)$

$H(\mathbf{r})=\sum_{i=1,2} \Psi_{i}^{\dagger}(\mathbf{r})\left(-\frac{\nabla^{2}}{2 m_{i}}\right) \Psi_{i}(\mathbf{r})+\sum_{i j} \frac{g_{i j}}{2} \Psi_{i}^{\dagger} \Psi_{j}^{\dagger} \Psi_{j} \Psi_{i}(\mathbf{r})$.

Here, $\mathbf{r}=(x, y, z)$ is the coordinate; $m_{i}$ and $\Psi_{i}$ are respectively the mass and field operator of boson species $i ; g_{i i}=$ $4 \pi a_{i i} / m_{i}$ and $g_{12}=2 \pi a_{12} / \mu\left[\mu=m_{1} m_{2} /\left(m_{1}+m_{2}\right)\right]$ are the intra- and interspecies couplings. Given the atoms confined uniformly within $z \in[-L, L]$ and under a periodic boundary condition, the momenta along $z$ are quantized as $k_{n}=n \pi / L$ $(n=0, \pm 1, \ldots)$. Based on the Bogoliubov theory for a homogeneous mixture with densities $n_{1}, n_{2}$ [29], we can get the LHY energy per volume as

$$
\begin{aligned}
\mathcal{E}_{\mathrm{LHY}}= & \int \frac{d^{2} \mathbf{q}}{2(2 \pi)^{2}} \frac{1}{2 L} \sum_{n}\left[E_{n \mathbf{q}}^{+}+E_{n \mathbf{q}}^{-}-\sum_{i=1,2}\left(\epsilon_{n \mathbf{q}}^{(i)}+g_{i i} n_{i}\right)\right] \\
& +\int \frac{d^{3} \mathbf{k}}{2(2 \pi)^{3}} \frac{m_{1} g_{11}^{2} n_{1}^{2}+m_{2} g_{22}^{2} n_{2}^{2}+4 \mu g_{12}^{2} n_{1} n_{2}}{\mathbf{k}^{2}} .
\end{aligned}
$$

Here, $\mathbf{q}$ and $\mathbf{k}$ are respectively $2 \mathrm{D}$ and $3 \mathrm{D}$ momentum vectors, and the quasiparticle energies read



with $\omega_{i}=\sqrt{\epsilon_{n \mathbf{q}}^{(i)}\left(\epsilon_{n \mathbf{q}}^{(i)}+2 g_{i i} n_{i}\right)} \quad$ and $\quad \epsilon_{n \mathbf{q}}^{(i)}=\left[(n \pi / L)^{2}+\right.$ $\left.\mathbf{q}^{2}\right] /\left(2 m_{i}\right)$. We note that the LHY energy in quasilow dimensions was studied previously with different techniques aiming at equal-mass mixtures [24,25]. In comparison, our scheme can apply for an arbitrary mass ratio. For the equal-mass case, we have checked that Eq. (1) can reproduce the LHY energy effectively in the 2D [24] or 3D [12] limit, given the boson densities are small or large.

To investigate the stability of a self-bound state, we have to go beyond the bulk description and employ a spatially varying ansatz $\Psi_{i}(\mathbf{r})$. Using the single-mode approximation $\Psi_{i}(\mathbf{r})=$ $\sqrt{N_{i}} \phi(\mathbf{r})$, we get the energy functional

$$
E=E_{\text {kin }}+E_{\mathrm{mf}}+E_{\mathrm{LHY}},
$$

with $\quad E_{\mathrm{kin}}=\sum_{i} N_{i} \int d \mathbf{r} \phi^{*}(\mathbf{r})\left(-\frac{\nabla^{2}}{2 m_{i}}\right) \phi(\mathbf{r}), \quad E_{\mathrm{mf}}=$ $\left(g_{11} N_{1}^{2} / 2+g_{22} N_{2}^{2} / 2+g_{12} N_{1} N_{2}\right) \int d \mathbf{r}|\phi(\mathbf{r})|^{4}$, and $E_{\mathrm{LHY}}=$ $\int d \mathbf{r} \mathcal{E}_{\mathrm{LHY}}\left[n_{i}(\mathbf{r})\right]$, where $n_{i}(\mathbf{r})=N_{i}\left|\phi_{i}(\mathbf{r})\right|^{2}$. We further assume the number ratio as $N_{1} / N_{2}=\sqrt{g_{22} / g_{11}}$ in order to minimize $E_{\mathrm{mf}}$ [12]. The above assumptions have been shown to well predict the liquid-gas transition in 3D droplets [8]. For the current case with a uniform trap under a periodic condition $\phi(\mathbf{r})=\phi\left(\mathbf{r}+2 L \mathbf{e}_{z}\right)$, we adopt an extended Gaussian-type ansatz as follows:

$\phi(\mathbf{r})=\frac{1}{\sqrt{\mathcal{N}}} \exp \left(-\frac{x^{2}+y^{2}}{2 \sigma_{x y}^{2}}\right)\left[\sum_{\nu=-\infty}^{\infty} \exp \left(-\frac{(z-2 \nu L)^{2}}{2 \sigma_{z}^{2}}\right)\right]$

Here, $\mathcal{N}$ is the normalization factor; $\sigma_{x y}$ and $\sigma_{z}$ are two variational parameters and represent, respectively, the sizes of the bound state along $x y$ and $z$. The ground state can be obtained by minimizing the energy functional (3) in terms of $\sigma_{x y}$ and $\sigma_{z}$.

In this Letter, we specifically consider the two hyperfine states of ${ }^{39} \mathrm{~K}$ atoms, $|1\rangle \equiv\left|F=1, m_{F}=0\right\rangle,|2\rangle \equiv \mid F=$ $\left.1, m_{F}=-1\right\rangle$, as have been well studied in 3D droplet experiments [8-10]. In this case, $a_{22}=35 a_{B}, a_{12}=-53 a_{B}\left(a_{B}\right.$ is the Bohr radius), and $a_{11}$ is highly tunable by a magnetic field. We will focus on the mean-field collapse regime with $\delta a \equiv a_{12}+\sqrt{a_{11} a_{22}}<0$ and study how the uniform confinement affects the quantum droplet. As we consider small $|\delta a|$ ( $\left.\ll a_{11}, a_{22},\left|a_{12}\right|\right)$, in calculating $E_{\mathrm{LHY}}$ we make the approximation $\delta a=0$ to avoid the phonon instability due to the complex spectrum (2). Other rectified theories on this have appeared recently [30-32]. Throughout this Letter, we choose the length unit as $l_{0}=1 \mu \mathrm{m}$ and the energy unit as $E_{0}=$ $1 /\left(2 m l_{0}^{2}\right)$, with mass $m \equiv m_{1}=m_{2}$ for ${ }^{39} \mathrm{~K}$ atoms.

Results. By searching for the energy minimum in terms of $\sigma_{x y}$ and $\sigma_{z}$, i.e., $\partial E / \partial \sigma_{x y, z}=0$ and $\partial^{2} E / \partial \sigma_{x y, z}^{2}>0$, we find two candidates for the ground state: One is with finite $\sigma_{x y}$ and finite $\sigma_{z}$, which is smoothly connected to the 3D droplet for large $L$ and is thus referred to as a droplet; the other is with finite $\sigma_{x y}$ and $\sigma_{z} \rightarrow \infty$, which exists only under confinement and is referred to as a soliton. Different from the free-space case, here no gaseous ground state (both $\sigma_{x y, z} \rightarrow \infty$ ) can be found for finite $L$.

(I) Droplet solution. Figure 2 shows the droplet solution as varying $N$ at different $L$. One can see from Fig. 2(a1) that the total energy of the droplet continuously decreases as shrinking $L$, which can be attributed to the reduced kinetic and LHY energies, as shown by $E_{\text {kin }}$ and $E_{\text {LHY }}$ in Fig. 2(a2). The reduction of $E_{\text {kin }}$ is due to the enlarged energy gap and thus the suppressed excitation along $z$, while the reduction of $E_{\mathrm{LHY}}$ here is consistent with that found in Refs. [24,25]. Another remarkable effect of finite $L$ is that, now the droplet only survives within a finite number window $\left[N_{d 1}, N_{d 2}\right]$, unlike the free-space droplet that just requires a lower number bound. This number window becomes narrower for smaller $L$, due to the existence of another competitive bound state (soliton, as discussed later). In particular, we see that a small $L$ also gives rise to a small upper bound $N_{d 2}$, which is consistent with the boundary effect as illustrated in Fig. 1.

Figures 2(b1) and 2(b2) show both $\sigma_{x y}$ and $\sigma_{z}$ evolving nonmonotonically with $N$. Near the vanishing point of the 

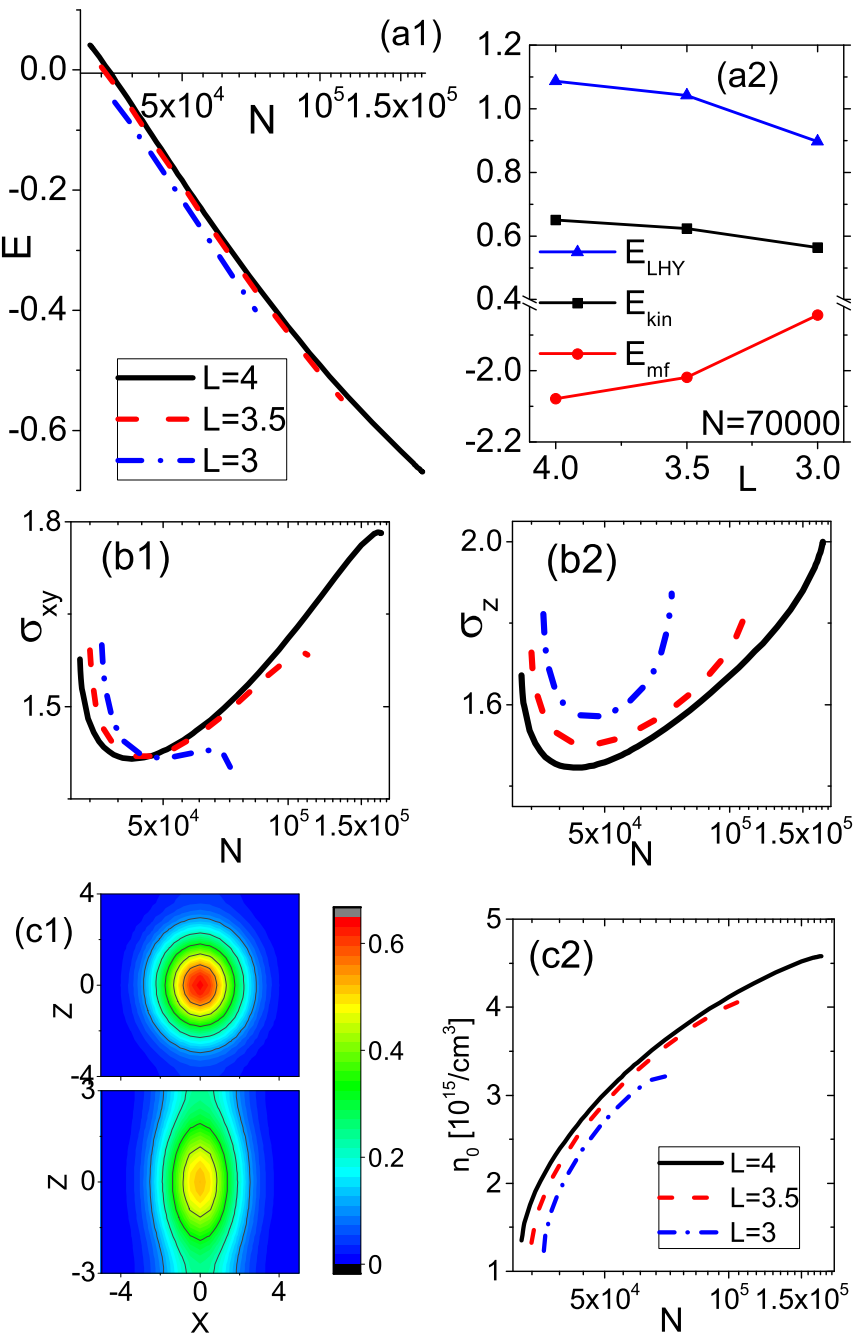

FIG. 2. Droplet state of ${ }^{39} \mathrm{~K}$ atoms in Q2D with $\delta a=-5 a_{B}$. (a1) Droplet energy $E$ as a function of atom number $N$ at different $L=$ 4, 3.5, 3. (a2) $E_{\mathrm{LHY}}, E_{\mathrm{kin}}$, and $E_{\mathrm{mf}}$ at different $L$ for a given $N=7 \times$ $10^{4}$. (b1), (b2) Droplet sizes $\sigma_{x y}$ and $\sigma_{z}$ as functions of $N$ at various $L$ [with the same line style as in (a1)]. (c1) Contour plot of droplet wave function $\phi(\mathbf{r})$ in the $(x, z)$ plane (with $y=0$ ) for a given $N=7 \times 10^{4}$ at $L=4$ (upper panel) and 3 (lower panel). (c2) Peak density $n_{0}$ (in units of $10^{15} / \mathrm{cm}^{3}$ ) as a function of $N$. Here, the length and energy units are respectively $l_{0}=1 \mu \mathrm{m}$ and $E_{0}=1 /\left(2 m l_{0}^{2}\right)$.

droplet $\left(N \sim N_{d 2}\right.$ ), shrinking $L$ will lead to a smaller $\sigma_{x y}$ but a larger $\sigma_{z}$. This means that by tightening the confinement, the droplet wave function will change from isotropic to a highly elongated shape (along $z$ ), as shown in Fig. 2(c1). This counterintuitive change shares the same reason with the suppressed $E_{\text {kin }}$ as shown in Fig. 2(a2), i.e., the lower $E_{\text {kin }}$ corresponds to an extended density distribution along $z$. Because of such an extended distribution, the peak density of the droplet $n_{0}$ also decreases as $L$ shrinks [see Fig. 2(c2)]. Here, $n_{0} \sim 10^{15} / \mathrm{cm}^{3}$, the same order as the typical density in the 3D droplet experiment [10].

(II) Soliton solution. The uniform trap along $z$ can also support a soliton bound state $(E<0)$ at $\sigma_{z}=\infty$ and a finite $\sigma_{x y}$, where the density modulation is allowed only along free $(x y)$ directions but not along the confined $(z)$ direction.

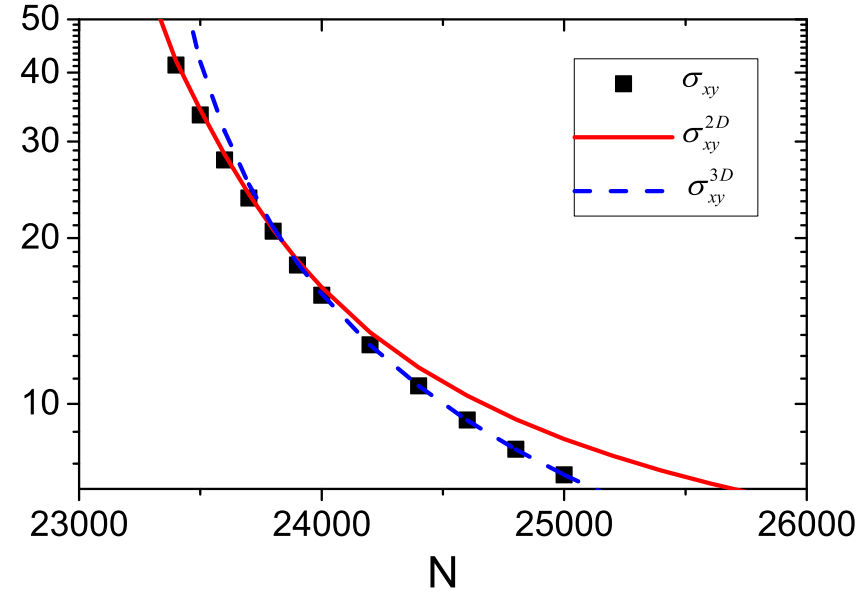

FIG. 3. Transverse size of the soliton state $\sigma_{x y}$ (black square) for ${ }^{39} \mathrm{~K}$ atoms in Q2D. Here, $\delta a=-5 a_{B}$ and $L=3$. Red solid and blue dashed lines are the fits to results in $2 \mathrm{D}$ and $3 \mathrm{D}$ limits (see text). The length unit is $l_{0}=1 \mu \mathrm{m}$.

Different from the Q1D soliton [26-28], here only the kinetic and mean-field terms are inadequate to support the Q2D soliton. For instance, in the 2D limit we have $E_{\text {kin }} \sim \sigma_{x y}^{-2}$ and $E_{\mathrm{mf}} \sim-L^{-1} \sigma_{x y}^{-2}$, and one has to incorporate the contribution from $E_{\mathrm{LHY}}$ to enable an energy minimum at finite $\sigma_{x y}$. It is noted that such an LHY-stabilized soliton in the 2D limit is equivalent to the 2D droplet studied in Ref. [21].

In Fig. 3 we show the soliton size $\sigma_{x y}$ as varying $N$ at fixed $L=3 \mu \mathrm{m}$. To analyze its behavior in the limits of small and large $N$, we utilize the analytical expressions of $\epsilon_{\mathrm{LHY}}$ in $2 \mathrm{D}$ and 3D limits [same as Eq. (7) in Ref. [24] and Eq. (5) in Ref. [12]], and obtain the integrated LHY energy as

$$
\begin{gathered}
E_{\mathrm{LHY}}^{2 \mathrm{D}}=\frac{2 D^{2}}{m \sigma_{x y}^{2}}\left(\ln \left[\frac{4 L D^{1 / 2}}{\sigma_{x y}}\right]+\frac{8 L^{2}}{9 \sigma_{x y}^{2}} D\right), \\
E_{\mathrm{LHY}}^{3 \mathrm{D}}=\frac{1024 L}{75 \pi m \sigma_{x y}^{3}} D^{5 / 2},
\end{gathered}
$$

with $D=\left(N_{1} a_{11}+N_{2} a_{22}\right) /(2 L)$. Minimizing the total energy $E$, we can obtain the equilibrium size, $\sigma_{x y}^{2 \mathrm{D}}$ or $\sigma_{x y}^{3 \mathrm{D}}$, in the $2 \mathrm{D}$ or 3D limit. Figure 3 shows that $\sigma_{x y}^{2 \mathrm{D}}\left(\sigma_{x y}^{3 \mathrm{D}}\right)$ fits well to the soliton size $\sigma_{x y}$ in a small (large) $N$ limit.

(III) Droplet-soliton transition and coexistence. After identifying the individual property of the droplet and soliton, now we turn to their competition. In Fig. 4, we show their transition and coexistence as varying $N$ for a fixed $L=3.5 \mu \mathrm{m}$. As seen from Fig. 4(a), the energies of the droplet and soliton cross twice as increasing $N$, which gives two transition points respectively at $N_{c 1}$ and $N_{c 2}$. Their individual stability and mutual competition can be clearly seen from the energy contour plots $E\left(\sigma_{x y}, \sigma_{z}\right)$ in Figs. 4(c1)-4(c5), together with the comparison of their transverse sizes $\sigma_{x y}$ shown in Fig. 4(b).

For small $N$, the only energy minimum represents a soliton state, i.e., at $\sigma_{z} \rightarrow \infty$ and a finite $\sigma_{x y}$ [see Fig. 4(c1)]. As increasing $N$ to $N_{d 1}$, the droplet starts to emerge as an additional energy minimum at finite $\sigma_{z}$ and a smaller $\sigma_{x y}$ [Fig. 4(b)]. The double minima reach the same energy at the first transition point $N_{c 1}$ [Fig. 4(c2)]. 


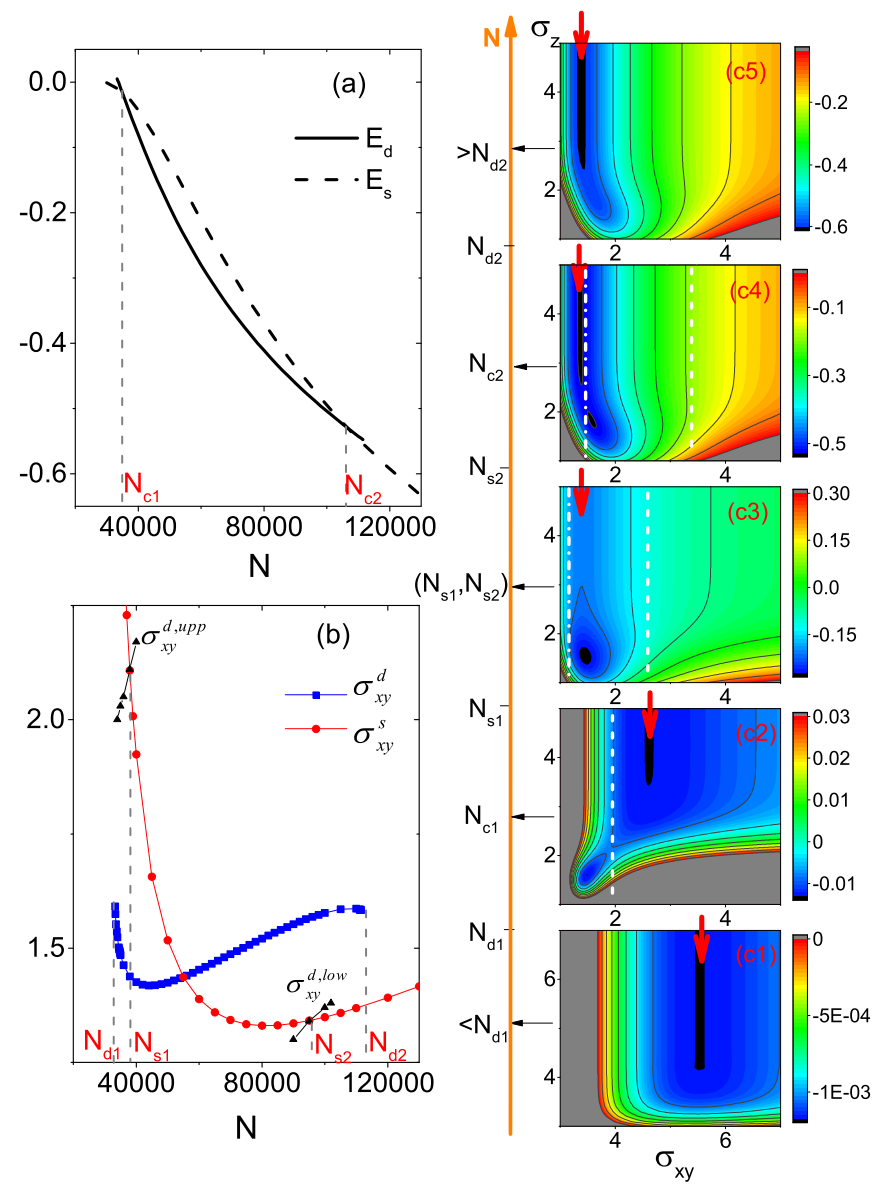

FIG. 4. Droplet-soliton transition and coexistence at $\delta a=-5 a_{B}$ and $L=3.5 \mu \mathrm{m}$. (a) Energies of a droplet $\left(E_{d}\right)$ and soliton $\left(E_{s}\right)$ as functions of $N$. The energy crossings determine two transition points at $N_{c 1}$ and $N_{c 2}$. (b) Transverse sizes of a droplet $\left(\sigma_{x y}^{d}\right)$ and soliton

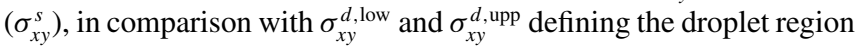
(see text). The droplet is locally stable for $N \in\left(N_{d 1}, N_{d 2}\right)$, and is the only stable (ground) state for $N \in\left(N_{s 1}, N_{s 2}\right)$ when the soliton enters the droplet region. Droplet-soliton coexistence occurs at $N \in$ $\left(N_{d 1}, N_{s 1}\right) \bigcup\left(N_{s 2}, N_{d 2}\right)$. (c1)-(c5) Contour plot of $E\left(\sigma_{x y}, \sigma_{z}\right)$ for various $N\left(10^{4}\right)$ : (c1) 3, (c2) 3.48(= $\left.N_{c 1}\right)$, (c3) 6, (c4) 10.66(= $\left.N_{c 2}\right)$, (c5) 11.5. The white dashed-dotted and dashed lines mark the locations of $\sigma_{x y}^{d, \text { low }}$ and $\sigma_{x y}^{d \text {,upp }}$, and the red arrows mark $\sigma_{x y}^{s}$. The length and energy units are the same as in Fig. 2.

To facilitate later discussions, let us define the droplet region in the energy landscape along $\sigma_{x y}$, with a lower bound $\sigma_{x y}^{d \text {, low }}$ and upper bound $\sigma_{x y}^{d \text {,upp }}$ [marked by the dashed-dotted and dashed lines in Figs. 4(c2)-4(c4)]. Within this region, for any $\sigma_{x y} \in\left(\sigma_{x y}^{d, \text { low }}, \sigma_{x y}^{d \text {,upp }}\right)$, the energy minimum occurs at a finite $\sigma_{z}$. By this definition, the droplet solution stays right within the droplet region [see Figs. 4(c2)-4(c4)]. Then, if the soliton solution also lies in this region [see the red arrow in Fig. 4(c3)], i.e., when $\sigma_{x y}^{s} \in\left(\sigma_{x y}^{d, \text { low }}, \sigma_{x y}^{d \text {, upp }}\right)$, the soliton will become locally unstable and flow from $\sigma_{z}=\infty$ to the droplet minimum. In Fig. 4(b), we denote the atom number at the intersection of $\sigma_{x y}^{s}$ and $\sigma_{x y}^{d \text {,upp }}\left(\sigma_{x y}^{d \text { low }}\right)$ as $N_{s 1}\left(N_{s 2}\right)$. Correspondingly, when $N \in\left[N_{s 1}, N_{s 2}\right]$, the droplet is the only stable (ground) state [see Fig. 4(c3)]. For $N$ beyond $N_{s 2}$, the soliton

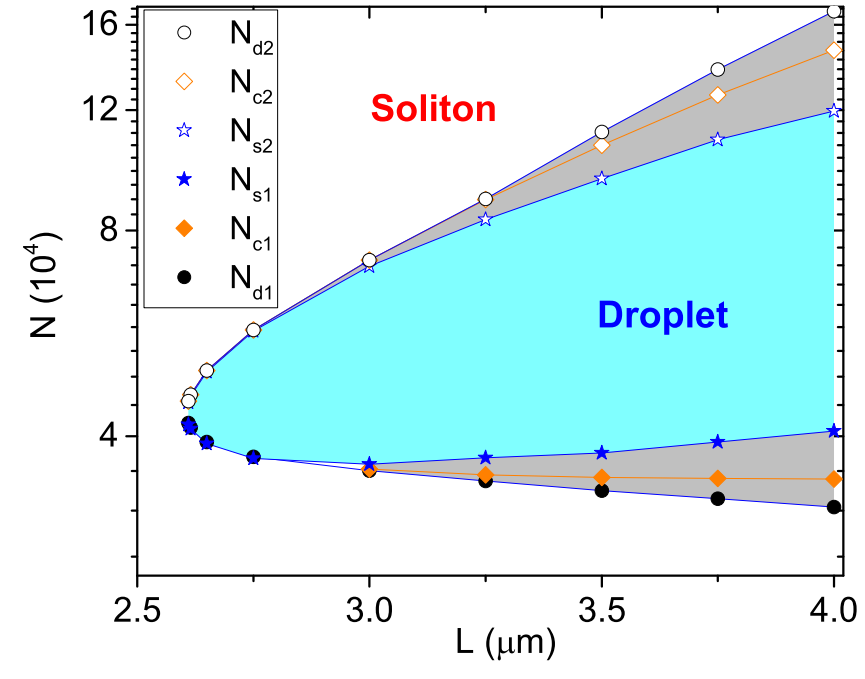

FIG. 5. Phase diagram in the $(N, L)$ plane for a ${ }^{39} \mathrm{~K}$ mixture at $\delta a=-5 a_{B}$. The droplet, soliton, and their coexistence regions are respectively shown by blue, white, and gray colors. Their phase boundaries are given by $N_{d 1}, N_{s 1}, N_{s 2}$, and $N_{d 2}$ (see text). Dropletsoliton transitions (energy crossing) occur at $N_{c 1}$ and $N_{c 2}$, denoted by solid and open orange diamonds.

moves outside the droplet region and they can coexist again. Their second transition occurs at $N_{c 2}$ when the two minima have the same energy [see Fig. 4(c4)]. The coexistence stops at $N=N_{d 2}$ when the droplet solution disappears, and for $N>N_{d 2}$ the only stable state becomes a soliton again [see Fig. 4(c5)].

From above, we can see that the droplet-soliton competition is most pronounced when the soliton enters the droplet region, or equivalently, when they have similar sizes along free $(x y)$ directions. On the other hand, the instability of a droplet as well as the reentrance of a soliton at large $N>N_{d 2}$ can be attributed to the boundary effect (Fig. 1), when the droplet size along $z$ is comparable with $L$. For instance, at $N_{d 2}$ we have $\sigma_{z}=1.86 \mu \mathrm{m}$, beyond half of $L(=3.5 \mu \mathrm{m})$.

(IV) Phase diagram. To fully explore the confinement effect, we have carried out a similar analysis for different $L$ and arrived at the phase diagram in the $(N, L)$ plane as shown in Fig. 5. One can see that the droplet state (blue color) only survives within a finite number window that sensitively depends on the value of $L$. It will give way to the soliton state (white color) for very large or small $N$, or for small $L$. Near their transition points $N_{c 1}$ and $N_{c 2}$ (orange diamonds), the droplet and soliton can coexist with each other, and their coexistence region (gray color) also depends sensitively on $L$.

In fact, for $L \in(2.6,3) \mu \mathrm{m}$ we find continuous transitions between the droplet and soliton, i.e., the location of the energy minimum continuously changes between finite and infinite $\sigma_{z}$ across the phase boundaries, For $L<2.6 \mu \mathrm{m}$, no droplet solution can be found and the soliton is the only stable (ground) state. Again this can be attributed to the large energy gap along $z$, which rules out the possibility of density modulation in this direction.

Discussion. In this Letter, we have adopted the local density approximation (LDA) to compute $E_{\mathrm{LHY}}$, which was shown to predict the 3D droplets quantitatively well $[8,10]$. 
Here, we remark that the LDA is even more qualified in our case, especially along the confined direction with small $L$. This is because as reducing $L$, the density distribution gets more extended along $z$ and the kinetic energy is further suppressed [Figs. 2(a2) and 2(c1)]. In fact, we have $\eta_{z} \equiv$ $E_{\mathrm{kin}, \mathrm{Z}} / E_{\mathrm{LHY}} \ll 1$ in a broad parameter regime considered in this Letter. Taking the case in Fig. 4, for instance, the ratio $\eta_{z}$ is 0.46 at $N_{c 1}$ and gets even smaller to 0.08 at $N_{c 2}$. This is to say, the typical length at which the density varies is visibly longer than that characterizing the LHY correction, which justifies the use of LDA in our setup.

Though we have taken the periodic boundary condition, our results shed important light on the hard-wall boundary case as realized in current experiments [33-37]. We expect the hard-wall boundary can equally cause the instability of a quantum droplet at small $L$ or large $N$ (see Fig. 1). Nevertheless, in this case the droplet cannot extend outside the boundary, in contrast with the periodic case [see Fig. 2(c1)], and therefore the actual phase diagram needs to be reexamined. Finally, it is worth pointing out that the boundary effect here is unlikely to apply for harmonic confinements, where the boundary cannot be clearly defined and the eigenmode is also different. This follows that the physics near $N_{c 2}$, as mostly driven by the boundary effect, would disappear for harmonically confined systems. This expectation is consistent with the recent experiment of harmonically trapped BoseBose mixtures in Q1D [9], where only one droplet-soliton transition (corresponding to $N_{c 1}$ in this Letter) was observed.

Acknowledgments. The work is supported by the National Key Research and Development Program of China (2018YFA0307600, 2016YFA0300603), the National Natural Science Foundation of China (No. 12074419), and the Strategic Priority Research Program of Chinese Academy of Sciences (No. XDB33000000).
[1] I. Ferrier-Barbut, H. Kadau, M. Schmitt, M. Wenzel, and T. Pfau, Observation of Quantum Droplets in a Strongly Dipolar Bose Gas, Phys. Rev. Lett. 116, 215301 (2016).

[2] M. Schmitt, M. Wenzel, F. Böttcher, I. Ferrier-Barbut, and T. Pfau, Self-bound droplets of a dilute magnetic quantum liquid, Nature (London) 539, 259 (2016).

[3] I. Ferrier-Barbut, M. Schmitt, M. Wenzel, H. Kadau, and T. Pfau, Liquid quantum droplets of ultracold magnetic atoms, J. Phys. B: At., Mol. Opt. Phys. 49, 214004 (2016).

[4] L. Chomaz, S. Baier, D. Petter, M. J. Mark, F. Wächtler, L. Santos, and F. Ferlaino, Quantum-Fluctuation-Driven Crossover from a Dilute Bose-Einstein Condensate to a Macrodroplet in a Dipolar Quantum Fluid, Phys. Rev. X 6, 041039 (2016).

[5] L. Tanzi, E. Lucioni, F. Famà, J. Catani, A. Fioretti, C. Gabbanini, R. N. Bisset, L. Santos, and G. Modugno, Observation of a Dipolar Quantum Gas with Metastable Supersolid Properties, Phys. Rev. Lett. 122, 130405 (2019).

[6] F. Böttcher, J.-N. Schmidt, M. Wenzel, J. Hertkorn, M. Guo, T. Langen, and T. Pfau, Transient Supersolid Properties in an Array of Dipolar Quantum Droplets, Phys. Rev. X 9, 011051 (2019).

[7] L. Chomaz, D. Petter, P. Ilzhöfer, G. Natale, A. Trautmann, C. Politi, G. Durastante, R. M. W. van Bijnen, A. Patscheider, M. Sohmen, M. J. Mark, and F. Ferlaino, Long-Lived and Transient Supersolid Behaviors in Dipolar Quantum Gases, Phys. Rev. X 9, 021012 (2019).

[8] C. R. Cabrera, L. Tanzi, J. Sanz, B. Naylor, P. Thomas, P. Cheiney, and L. Tarruell, Quantum liquid droplets in a mixture of Bose-Einstein condensates, Science 359, 301 (2018).

[9] P. Cheiney, C. R. Cabrera, J. Sanz, B. Naylor, L. Tanzi, and L. Tarruell, Bright Soliton to Quantum Droplet Transition in a Mixture of Bose-Einstein Condensates, Phys. Rev. Lett. 120, 135301 (2018).

[10] G. Semeghini, G. Ferioli, L. Masi, C. Mazzinghi, L. Wolswijk, F. Minardi, M. Modugno, G. Modugno, M. Inguscio, and M. Fattori, Self-Bound Quantum Droplets of Atomic Mixtures in Free Space, Phys. Rev. Lett. 120, 235301 (2018).
[11] C. D’Errico, A. Burchianti, M. Prevedelli, L. Salasnich, F. Ancilotto, M. Modugno, F. Minardi, and C. Fort, Observation of quantum droplets in a heteronuclear bosonic mixture, Phys. Rev. Research 1, 033155 (2019).

[12] D. S. Petrov, Quantum Mechanical Stabilization of a Collapsing Bose-Bose Mixture, Phys. Rev. Lett. 115, 155302 (2015).

[13] X. Cui, Spin-orbit-coupling-induced quantum droplet in ultracold Bose-Fermi mixtures, Phys. Rev. A 98, 023630 (2018).

[14] S. Adhikari, A self-bound matter-wave boson-fermion quantum ball, Laser Phys. Lett. 15, 095501 (2018).

[15] D. Rakshit, T. Karpiuk, M. Brewczyk, and M. Gajda, Quantum Bose-Fermi droplets, SciPost Phys. 6, 079 (2019).

[16] D. Rakshit, T. Karpiuk, P. Zin, M. Brewczyk, M. Lewenstein, and M. Gajda, Self-bound Bose-Fermi liquids in lower dimensions, New J. Phys. 21, 073027 (2019).

[17] M. Wenzel, T. Pfau, and I. Ferrier-Barbut, A fermionic impurity in a dipolar quantum droplet, Phys. Scr. 93, 104004 (2018).

[18] J.-B. Wang, J.-S. Pan, X. Cui, and W. Yi, Quantum droplets in a mixture of Bose-Fermi superfluids, Chin. Phys. Lett. 37, 076701 (2020).

[19] J. C. Smith, D. Baillie, and P. B. Blakie, Quantum Droplet States of a Binary Magnetic Gas, Phys. Rev. Lett. 126, 025302 (2021).

[20] R. N. Bisset, L. A. Peña Ardila, and L. Santos, Quantum Droplets of Dipolar Mixtures, Phys. Rev. Lett. 126, 025301 (2021).

[21] D. S. Petrov and G. E. Astrakharchik, Ultradilute LowDimensional Liquids, Phys. Rev. Lett. 117, 100401 (2016).

[22] D. Edler, C. Mishra, F. Wächtler, R. Nath, S. Sinha, and L. Santos, Quantum Fluctuations in Quasi-One-Dimensional Dipolar Bose-Einstein Condensates, Phys. Rev. Lett. 119, 050403 (2017).

[23] K. Jachymski and R. Oldziejewski, Nonuniversal beyondmean-field properties of quasi-two-dimensional dipolar Bose gases, Phys. Rev. A 98, 043601 (2018).

[24] P. Zin, M. Pylak, T. Wasak, M. Gajda, and Z. Idziaszek, Quantum Bose-Bose droplets at a dimensional crossover, Phys. Rev. A 98, 051603(R) (2018). 
[25] T. Ilg, J. Kumlin, L. Santos, D. S. Petrov, and H. P. Büchler, Dimensional crossover for the beyond-mean-field correction in Bose gases, Phys. Rev. A 98, 051604(R) (2018).

[26] V. M. Pérez-García, H. Michinel, and H. Herrero, Bose-Einstein solitons in highly asymmetric traps, Phys. Rev. A 57, 3837 (1998).

[27] K. E. Strecker, G. B. Partridge, A. G. Truscott, and R. G. Hulet, Formation and propagation of matter-wave soliton trains, Nature (London) 417, 150 (2002).

[28] L. Khaykovich, F. Schreck, G. Ferrari, T. Bourdel, J. Cubizolles, L. D. Carr, Y. Castin, and C. Salomon, Formation of a matterwave bright soliton, Science 296, 1290 (2002).

[29] C. J. Pethick and H. Smith, Bose-Einstein Condensation in Dilute Gases (Cambridge University Press, Cambridge, U.K., 2002).

[30] H. Hu and X.-J. Liu, Consistent Theory of Self-Bound Quantum Droplets with Bosonic Pairing, Phys. Rev. Lett. 125, 195302 (2020).

[31] M. Ota and G. E. Astrakharchik, Beyond Lee-Huang-Yang description of self-bound Bose mixtures, SciPost Phys. 9, 020 (2020).
[32] Q. Gu and L. Yin, Phonon stability and sound velocity of quantum droplets in a boson mixture, Phys. Rev. B 102, 220503(R) (2020).

[33] A. L. Gaunt, T. F. Schmidutz, I. Gotlibovych, R. P. Smith, and Z. Hadzibabic, Bose-Einstein Condensation of Atoms in a Uniform Potential, Phys. Rev. Lett. 110, 200406 (2013).

[34] I. Gotlibovych, T. F. Schmidutz, A. L. Gaunt, N. Navon, R. P. Smith, and Z. Hadzibabic, Observing properties of an interacting homogeneous Bose-Einstein condensate: Heisenberg-limited momentum spread, interaction energy, and free-expansion dynamics, Phys. Rev. A 89, 061604(R) (2014).

[35] C. Eigen, A. L. Gaunt, A. Suleymanzade, N. Navon, Z. Hadzibabic, and R. P. Smith, Observation of Weak Collapse in a Bose-Einstein Condensate, Phys. Rev. X 6, 041058 (2016).

[36] B. Mukherjee, Z. Yan, P. B. Patel, Z. Hadzibabic, T. Yefsah, J. Struck, and M. W. Zwierlein, Homogeneous Atomic Fermi Gases, Phys. Rev. Lett. 118, 123401 (2017).

[37] K. Hueck, N. Luick, L. Sobirey, J. Siegl, T. Lompe, and H. Moritz, Two-Dimensional Homogeneous Fermi Gases, Phys. Rev. Lett. 120, 060402 (2018). 